\title{
Genetic analysis of genes causing hypertension and stroke in spontaneously hypertensive rats
}

\author{
HIDEYUKI YAMAMOTO $^{1 *}$, DAISUKE OKUZAKI ${ }^{3 *}$, KYOSUKE YAMANISHI $^{2}$, YUNFENG XU $^{1}$, \\ YUKO WATANABE ${ }^{4,5}$, MOMOKO YOSHIDA ${ }^{4,5}$, AKIFUMI YAMASHITA ${ }^{4}$, NAOHISA GOTO ${ }^{4}$, \\ SEIJI NISHIGUCHI ${ }^{5}$, KAZUNORI SHIMADA ${ }^{5}$, HIROSHI NOJIMA ${ }^{3}$, TERUO YASUNAGA ${ }^{4}$, \\ HARUKI OKAMURA $^{1}$, HISATO MATSUNAGA ${ }^{2}$ and HIROMICHI YAMANISHI ${ }^{5}$ \\ ${ }^{1}$ Institute for Advanced Medical Sciences and ${ }^{2}$ Department of Neuropsychiatry, Hyogo College of Medicine, \\ Nishinomiya, Hyogo 663-8501; ${ }^{3}$ DNA-Chip Development Center for Infectious Diseases and \\ ${ }^{4}$ Department of Genome Informatics, Research Institute for Microbial Diseases, Osaka University, Suita, \\ Osaka 565-0871; ${ }^{5}$ Hirakata General Hospital for Developmental Disorders, Hirakata, Osaka 573-0122, Japan
}

Received December 15, 2012; Accepted February 22, 2013

DOI: $10.3892 /$ ijmm.2013.1304

\begin{abstract}
Spontaneously hypertensive rats (SHR) and strokeprone SHR (SHRSP) are frequently used as model rats not only in studies of essential hypertension and stroke, but also in studies of attention deficit hyperactivity disorder (ADHD). Normotensive Wistar-Kyoto rats (WKY) are normally used as controls in these studies. In this study, using these rats, we aimed to identify the genes causing hypertension and stroke, as well as the genes involved in ADHD. Since adrenal gland products can directly influence cardiovascular, endocrine and sympathetic nervous system functions, gene expression profiles in the adrenal glands of the 3 rat strains were examined using genome-wide microarray technology when the rats were 3 and 6 weeks of age, a period in which the rats are considered to be in a pre-hypertensive state. Gene expression profiles were compared between SHR and WKY and between SHRSP and SHR. A total of 353 genes showing more than a 4-fold increase
\end{abstract}

Correspondence to: Dr Hiromichi Yamanishi, Hirakata General Hospital for Developmental Disorders, 2-1-1 Tsudahigashi, Hirakata, Osaka 573-0122, Japan

E-mail: hirochan@hirakataryoiku-med.or.jp

*Contributed equally

Abbreviations: ADHD, attention deficit hyperactivity disorder; BP, blood pressure; DAVID, Database for Annotation, Visualization and Integrated Discovery; GEO, Gene Expression Omnibus; GO, Gene Ontology; IPA, Ingenuity Pathway Analysis; SHR, spontaneously hypertensive rats; SHRSP, stroke-prone SHR; WKY, Wistar-Kyoto rats

Key words: attention deficit hyperactivity disorder, adrenal gland, gene expression profiles, hypertension, microarray, spontaneously hypertensive rats, stroke-prone, normotensive Wistar-Kyoto rats or less than a 4-fold decrease in expression were isolated and candidate genes were selected as significantly enriched genes. SHR-specific genes isolated when the rats were 3 weeks of age contained 12 enriched genes related to transcriptional regulatory activity and those isolated when the rats were 6 weeks of age contained 6 enriched genes related to the regulation of blood pressure. SHRSP-specific genes isolated when the rats were 3 weeks of age contained 4 enriched genes related to the regulation of blood pressure and those isolated when the rats were 6 weeks of age contained 4 enriched genes related to the response to steroid hormone stimulus. Ingenuity pathway analysis of enriched SHR-specific genes revealed that 2 transcriptional regulators, cAMP responsive element modulator (Crem) and Fos-like antigen 1 (Fosll), interact with blood pressure-regulating genes, such as neurotensin (Nts), apelin (Apln) and epoxide hydrolase 2, cytoplasmic (Ephx2). Similar analyses of SHRSP-specific genes revealed that angiotensinogen $(A g t)$, one of the blood pressure-regulating genes, plays pivotal roles among SHRSP-specific genes. Moreover, genes associated with ADHD, such as low density lipoprotein receptor $(L d l r)$ and $C r e m$, are discussed.

\section{Introduction}

The polygenic nature of hypertension has made it difficult to isolate genes involved in the genesis of this disease. Microarrays are a powerful tool for studying the genetics of hypertension as they facilitate the measurement of the expression of thousands of genes simultaneously. Since rodent models of human essential hypertension are ideal for microarray research, animal models of essential hypertension have been investigated using microarrays $(1,2)$.

In this study, we present a comparison of adrenal gland gene expression in 2 strains of hypertensive rats: spontaneously hypertensive rats (SHR) and a substrain derived from SHR, stroke-prone SHR (SHRSP) (3-5). SHR, the current paradigm for essential hypertension research, were developed in a breeding program based solely on selection by elevated blood 
pressure (BP) in Wistar rats (3). Normotensive descendants of Wistar-Kyoto rats (WKY), from which SHR were derived, were used as the controls $(3,4)$. SHRSP were established from SHR by selective inbreeding for stroke proneness (4).

Adrenal gland secretory products, both medullary and cortical, are logical candidates for the study of hypertension since they can directly influence cardiovascular, endocrine and sympathetic nervous system functions $(6,7)$. To our knowledge, this study represents the first attempt to compare the gene expression profiles of SHR and SHRSP in adrenal glands employing WKY as the controls, as early as 3 weeks of age. Since the first aim of this study was to identify candidate genes causing the transcription of BP-regulating genes in SHR, and the second aim was to identify genes involved in the genesis of stroke in SHRSP, we compared the gene expression profiles in the rats at 3 and 6 weeks of age, a period in which the rats are considered to be in a pre-hypertensive state, and isolated a total of 353 genes showing more than a 4-fold increase or less than a 4-fold decrease in expression.

After classifying all 353 genes according to their expression profiles, candidate genes were selected as significantly enriched genes using the Database for Annotation, Visualization and Integrated Discovery (DAVID) web tools $(8,9)$, and their interactions were analyzed with Ingenuity Pathway Analysis (IPA). Our analyses revealed that one of the SHR-specific transcriptional regulators, cAMP responsive element modulator ( $\mathrm{Crem}$ ), interacts, in the presence of Fos, with several BP-regulating genes, and suggested that one of the BP-regulating SHRSPspecific genes, angiotensinogen $(A g t)$, plays pivotal roles in symptoms associated with stroke. Since SHR and SHRSP are frequently used as animal models in studies of attention deficit hyperactivity disorder (ADHD), we examined the correlation between SHR- and SHRSP-specific genes and the characteristic symptoms of ADHD $(10,11)$.

\section{Materials and methods}

Animals. Animals, such as SHR/Izm, SHRSP/Izm, and WKY/Izm, were provided from the Disease Model Cooperative Research Association, Kyoto, Japan. Three-week-old rats were purchased and maintained for 2 days in our animal facility and were used as 3-week-old rats. Five-week-old rats were purchased and, after being maintained for 1 week in our animal facility, were used as 6-week-old rats. All these rats were euthanized by decapitation with a guillotine and, as soon as the rats were decapitated, the adrenal glands were extracted, cut into approximately 5- $\mathrm{mm}^{3}$ cubes, and stored in RNAlater (Ambion, Houston, TX, USA) at $-80^{\circ} \mathrm{C}$ until RNA extraction. The animals were handled with due care according to the guidelines established by the Japanese Association for Laboratory Animal Science, which comply with international rules and policies. All experiments involving rats were approved by the Animal Care and Use Committee of Hyogo College of Medicine on September 27, 2010.

RNA extraction. Total RNA of the entire adrenal glands was purified using an miRNeasy kit (Qiagen, Hilden, Germany) according to the manufacturer's instructions. Eluted RNAs were quantified using a NanoDrop ND-1000 version 3.5.2 spectrophotometer (Thermo Scientific, Wilmington, DE, USA).
RNA integrity was evaluated using an RNA 6000 LabChip kit and Bioanalyzer (Agilent Technologies, Inc., Santa Clara, CA, USA). Each RNA with RNA integrity numbers $>9.0$ was used for microarray experiments.

Microarray design. Expression profiling was generated using $4 \times 44 \mathrm{~K}$ whole rat genome oligo microarray version 3.0 G2519F (Agilent Technologies, Inc.). Each microarray uses 42,878 probes to interrogate 26,930 Entrez gene RNAs. Eighteen microarray analyses as 1 color experiment were performed with WKY, SHR and SHRSP at 3 and 6 weeks of age as biological triplicates. Each gene expression profile was compared between SHR and WKY and between SHRSP and SHR at 3 and 6 weeks of age.

Microarray analysis. Total RNA (200 ng) was reverse transcribed into double-stranded cDNA by AffinityScript multiple temperature reverse transcriptase and amplified for $2 \mathrm{~h}$ at $40^{\circ} \mathrm{C}$. The resulting cDNA was subsequently used for in vitro transcription by T7-polymerase and labeled with cyanine-3labeled cytosine triphosphate (Perkin-Elmer, Wellesley, MA, USA) for $2 \mathrm{~h}$ at $40^{\circ} \mathrm{C}$ using a Low Input Quick-Amp Labeling Kit (Agilent Technologies, Inc.) according to the manufacturer's instructions. After labeling, the rates of dye incorporation and quantification were measured using a NanoDrop ND-1000 version 3.5.2 spectrophotometer (Thermo Scientific) and were then fragmented for $30 \mathrm{~min}$ at $60^{\circ} \mathrm{C}$ in the dark. The labeled $1,650 \mathrm{ng}$ of each cRNA sample was then hybridized on Agilent $4 \times 44 \mathrm{~K}$ whole rat genome arrays (Agilent Design \#028282) at $65^{\circ} \mathrm{C}$ for $17 \mathrm{~h}$ with rotation in the dark. Hybridization was performed using a Gene Expression Hybridization kit (Agilent Technologies, Inc.) following the manufacturer's instructions. After washing in GE washing buffer, the slides were scanned with an Agilent Microarray Scanner (G2505C). Feature extraction software (version 10.5.1.1) employing defaults for all parameters was used to convert the images into gene expression data.

Microarray data analysis. Raw data were imported into Subio platform version 1.12 (Subio Inc., Aichi, Japan) for database management, quality control and statistical analysis. Raw intensity data were normalized to the 75 th percentile intensity of probes above background levels (gIsWellAbove=1). The normalized values were compared between SHR and WKY, and between SHRSP and SHR. SHR- and SHRSP-specific genes were defined to show signal ratios of a $>4.0$-fold increase or $<4.0$-fold decrease. We set the default cut-off value to $\mathrm{P}<0.01$ in this study. Raw data have been accepted in Gene Expression Omnibus (GEO), a public repository for microarray data, aimed at storing Minimum Information About Microarray Experiments (MIAME). Access to data concerning this study can be found under GEO experiment accession number GSE31457.

DAVID web tool analysis. An approach to annotation enrichment analysis was performed using DAVID web tools (version 6.7, 2010) $(8,9)$. This web-based resource provides a set of functional annotation tools for the statistical enrichment of genes categorized into Gene Ontology (GO) terms. We used the GO FAT category, which filters out very broad GO terms 
Table I. Number and classification of SHR- and SHRSP-specific probes compared between the 2 pairs of rat strains.

\begin{tabular}{|c|c|c|c|c|c|}
\hline \multirow[b]{2}{*}{ Classification } & \multicolumn{2}{|c|}{ SHR/WKY } & \multicolumn{2}{|c|}{ SHRSP/SHR } & \multirow[b]{2}{*}{ All } \\
\hline & $\begin{array}{c}\text { G-1 } \\
3 \text { weeks old }\end{array}$ & $\begin{array}{c}\mathrm{G}-2 \\
6 \text { weeks old }\end{array}$ & $\begin{array}{c}\text { G-3 } \\
3 \text { weeks old }\end{array}$ & $\begin{array}{c}\mathrm{G}-4 \\
6 \text { weeks old }\end{array}$ & \\
\hline All probes isolated & 123 & 165 & 44 & 75 & 407 \\
\hline Mapped probes & 108 & 151 & 43 & 73 & 375 \\
\hline Unmapped probes & 15 & 14 & 1 & 2 & 32 \\
\hline Identified unique genes & 101 & 143 & 42 & 67 & 353 \\
\hline Upregulated & 64 & 73 & 19 & 26 & 182 \\
\hline Downregulated & 37 & 70 & 23 & 41 & 171 \\
\hline Enriched GO terms & 1 & 8 & 4 & 2 & 15 \\
\hline Enriched genes & 12 & 42 & 17 & 9 & 80 \\
\hline
\end{tabular}

Number of SHR- and SHRSP-specific probes isolated from adrenal glands as described in the Materials and methods; 353 of the 407 isolated probes corresponded to unique genes having GenBank IDs. Using DAVID web tools, 353 genes categorized into GO terms and significantly enriched 15 GO terms, which included 80 enriched genes, were identified (Table II). SHR, spontaneously hypertensive rats; SHRSP, stroke-prone SHR; WKY, Wistar-Kyoto rats; GO, Gene Ontology.

to identify statistically enriched functional groups. Annotated gene and protein symbols are written in italics and regular font, respectively.

Ingenuity pathway analysis (IPA). IPA software (Ingenuity ${ }^{\circledR}$ Systems, http://www.ingenuity.com) was used for microarray analyses conducted to provide functionality for the interpretation of the gene expression data.IPA software, based on GO, biological processes, molecular function and genetic networks was used to map the biological correlation of differentially expressed genes into networks based on the published literature for each gene. The biological function network identifies biological functions and diseases that are most significant to the data set.

\section{Results}

Isolation and classification of SHR- and SHRSP-specific genes. We compared gene expression profiles between SHR and WKY and between SHRSP and SHR, at 3 and 6 weeks of age, and isolated SHR- and SHRSP-specific genes using genome-wide microarray technology. Since we expected that the expression of candidate genes was regulated long before the increase in BP, i.e., during the pre-hypertensive period, we examined the expression profiles of each probe using RNA samples prepared from adrenal glands obtained at 3 and 6 weeks of age, and isolated a total of 407 SHR- and SHRSPspecific probes showing a $>4$-fold increase or $<4$-fold decrease (Table I).

We classified 407 probes into 4 groups, from G-1 to G-4 (Table I). G-1 probes were isolated at 3 weeks of age and contained 123 SHR-specific probes. Their expression profiles were displayed as a heat map using Subio platform software (Fig. 1). These 123 probes corresponded to 101 unique genes, 64 of them showed a $>4$-fold increase and 37 showed $<4$-fold decrease (Table I). G-2 contained 143 SHR-specific genes isolated at 6 weeks of age, G-3 contained 42 SHRSP-specific genes isolated at 3 weeks of age, and G-4 contained 67 SHRSPspecific genes isolated at 6 weeks of age (Table I).
Categorization and enrichment of SHR- and SHRSP-specific genes. Using DAVID web tools, SHR- and SHRSP-specific genes were categorized into GO terms and significantly enriched genes were identified.

SHR-specific G-1 genes included 12 enriched genes categorized into one GO term, GO:0030528 (transcription regulator activity) (Table II, G-1). G-2 genes included 42 enriched genes and were categorized into $8 \mathrm{GO}$ terms. They included GO terms not only related to the circulatory system process, but also those related to the organic acid catabolic process, oxidation reduction and peptide receptor activity (Table II, G-2). These results suggest that enriched G-1 genes include candidate genes responsible for the genesis of hypertension in SHR.

SHRSP-specific G-3 genes included 17 enriched genes and were categorized into 4 GO terms (Table II, G-3), and G-4 genes included 9 enriched genes and were categorized into 2 GO terms, one of which was related to the control of steroid or fatty acid metabolism (Table II, G-4).

Interaction among SHR-specific genes isolated at 3 and 6 weeks of age. Eleven of the 12 enriched G-1 genes were upregulated and the remaining one, paired related homeobox protein-like 1 (Prrxl1), was downregulated (Table II, G-1). Since our results suggested the possibility that these 12 G-1 genes interact with G-2 genes, and since most of the 12 genes encode proteins related to RNA polymerase II transcription, we examined interactions among G-1 and G-2 genes by IPA and found 5 interactions (Fig. 2): i) tribbles homolog 3 (Drosophila) (Trib3) interacted with growth differentiation factor 15 (Gdf15); ii) peroxisome proliferator-activated receptor delta (Ppard) interacted with lecithin retinol acyltransferase (Lrat) and lipase, endothelial (Lipg); iii) Crem interacted with adrenergic, alpha-1D-, receptor (Adrald); iv) scleraxis $(S c x)$ interacted with secreted phosphoprotein 1 (Sppl); and v) fos-like antigen 1 (Fosll) interacted with neurotensin (Nts). However, we did not find any interactions between G-1 and BP-controlling G-2 genes, such as angiotensin II 

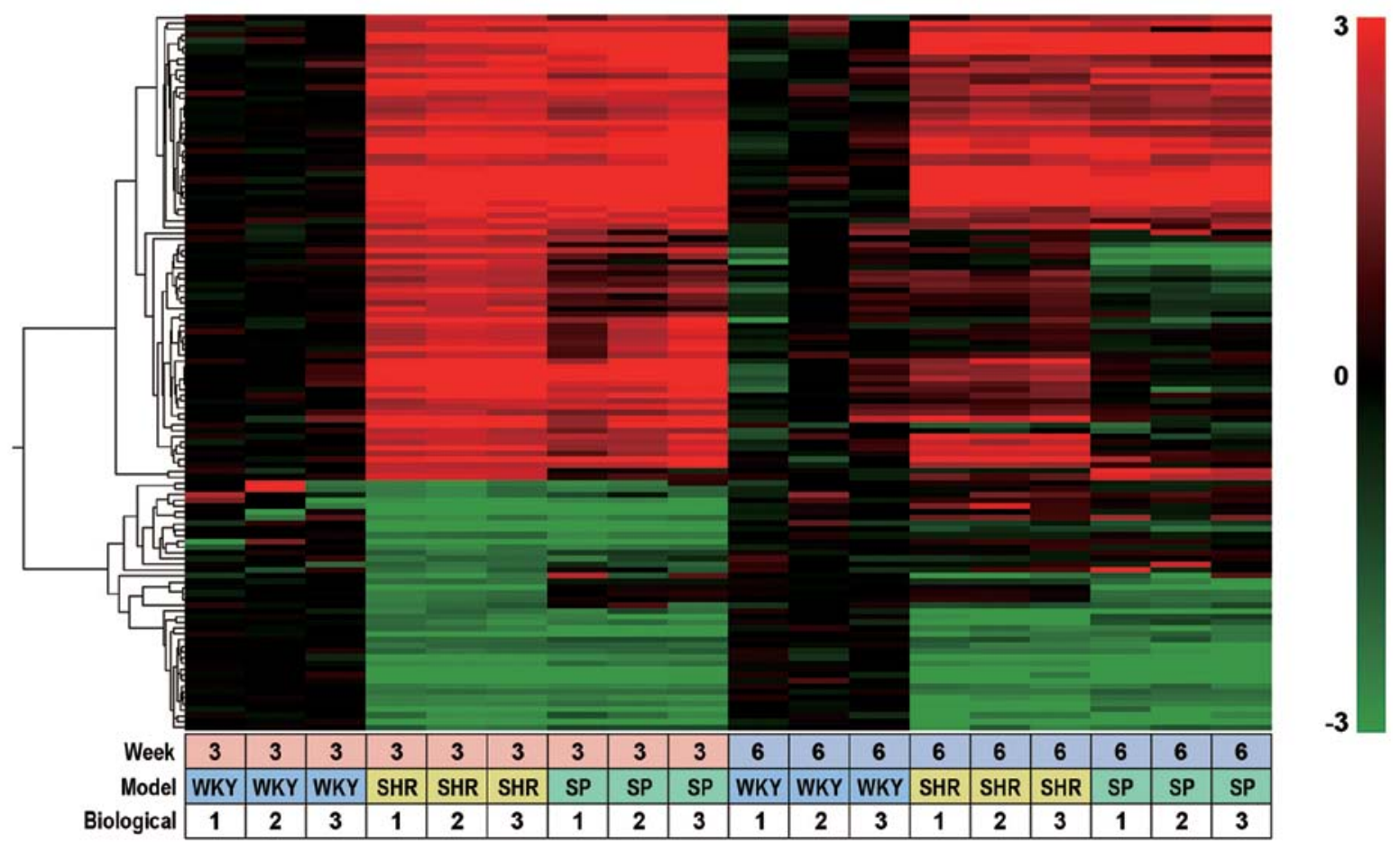

Figure 1. Heat map of SHR- and SHRSP-specific probes. Heat map of SHR- and SHRSP-specific probes isolated from the adrenal glands of rats at 3 and 6 weeks of age. Data were obtained with 407 probes of 3 rat strains, WKY, SHR, SHRSP, under 18 different experimental conditions (3 different rat strains, 2 different rat ages and triplicate experiments). Data obtained with G-1 probes, i.e., 123 out of 407 probes (Table I), were clustered based on their biological function and expression profiles using a hierarchical clustering program and Spearman's rank correlation. Values used for clustering were obtained by microarray experiments described in the Materials and methods. The color bar at the right side of the panel indicates the $\log _{2}$ ratio for each rat (SHR and SHRSP) at 3 or 6 weeks of age versus each rat (WKY) at 3 or 6 weeks of age. The bottom panel (small boxes) indicates the experimental conditions, i.e., examined at 3 or 6 weeks of age, 3 different rat strains and triplicate experiments. SHR, spontaneously hypertensive rats; SHRSP, stroke-prone SHR; WKY, Wistar-Kyoto rats.

receptor-associated protein (Agtrap), apelin (Apln), epoxide hydrolase 2, cytoplasmic (Ephx2) and urotensin 2 (Uts2) (Table II, G-2; GO:0003013, circulatory system process).

Interaction among SHRSP-specific genes isolated when the rats were 3 and 6 weeks of age. Since the study of enriched G-1 and G-2 genes suggested the possibility that enriched G-3 genes regulate the expression of G-4 genes isolated when the rats were 6 weeks of age, we examined interactions between G-3 and G-4 genes by IPA and found that Agt interacted not only with the 3 G-4 genes, hairy and enhancer of split 1 (Drosophila) (Hesl), low density lipoprotein receptor $(L d l r)$ and zinc finger and BTB domain containing 16 (Zbtb16), but also with 2 G-3 genes, Agtrap and heat shock 70 kDa protein 1B (Hspalb) (Fig. 3). We also found an interaction between $2 \mathrm{G}-4$ genes, Ldlr and insulin-induced gene 1 (Insig1) (Fig. 3).

\section{Discussion}

General considerations. We isolated 101 SHR-specific genes by comparing the gene expression profiles between SHR and WKY at 3 weeks of age and isolated 143 SHR-specific genes by comparing gene expression profiles of the rats at 6 weeks of age (Table I). Similarly, we isolated 42 SHRSP-specific genes by comparing the gene expression profiles between SHRSP and SHR at 3 weeks of age and isolated 67 SHRSP-specific genes by comparing the gene expression profiles of rats at 6 weeks of age (Table I). These results indicated that genetic differences between SHR and WKY were significantly larger than those between SHRSP and SHR.

Since SHR and SHRSP are frequently used as model rats, not only in studies of hypertension and stroke, but also in studies of ADHD $(10,11)$, these SHR- and SHRSP-specific genes are expected to include genes related to ADHD. These points are discussed later in this section.

SHR-specific genes possibly triggering hypertension in SHR. We found the following 5 interactions between G-1 and G-2 genes (Fig. 2): i) Trib3 interacted with Gdf15, which is known as a protective factor in response to cardiovascular injury $(12,13)$; ii) Ppard interacted with Lrat and Lipg, where the former is related to steroid metabolic process (14) and the latter is involved in lipoprotein metabolism and vascular biology (15); iii) Crem interacted with Adrald, which participates in norepinephrineepinephrine vasoconstriction (16); iv) $S c x$ interacted with $S p p 1$, which can act as a cytokine to stimulate lymphocyte immunoglobulin production $(17,18)$; and v) Fosll interacted with Nts, which encodes a precursor protein for both peptides (19) and participates in BP control by regulating blood vessel size (20). All these results suggest the possibility that the Trib3, Ppard, Crem, Scx and Fosll genes participate in the regulation of BP. However, all these interactions are not sufficient to explain the control of G-2 genes, such as Apln, Ephx2, Uts2 and Agtrap by G-1 genes (Table II, G-2, GO:0003013).

In order to identify further interactions between G-1 and G-2 genes, we suggested the presence of a gene that helps in 
Table II. Classification and enrichment of SHR- and SHRSP-specific genes.

\begin{tabular}{|c|c|c|c|c|c|c|}
\hline Group & GO category & GenBank ID & Description & GS & FC & P-value \\
\hline \multirow[t]{12}{*}{ G-1 } & GO:0030528 (P=0.006) & NM_172047 & ELL associated factor 2 & Eaf2 & 4.8 & 0.004 \\
\hline & \multirow[t]{11}{*}{$\begin{array}{l}\text { transcriptional regulator } \\
\text { activity }\end{array}$} & XM_226624 & $\begin{array}{l}\text { Elongation factor RNA polymerase II } 2 \text {, } \\
\text { transcript variant } 2\end{array}$ & Ell2 & 12.2 & 0.004 \\
\hline & & NM_031628 & $\begin{array}{l}\text { Nuclear receptor subfamily } 4 \text {, group A, member } 3 \text {, } \\
\text { transcript variant } 1\end{array}$ & $\mathrm{Nr} 4 \mathrm{a} 3$ & 7.9 & 0.005 \\
\hline & & NM_001130508 & Scleraxis & Scx & 7.4 & 0.008 \\
\hline & & NM_013141 & Peroxisome proliferator-activated receptor delta & Ppard & 6.2 & 0.004 \\
\hline & & NM_024385 & Hematopoietically expressed homeobox & Hhex & 5.3 & 0.001 \\
\hline & & NM_012953 & Fos-like antigen 1 & Fos11 & 31.7 & 0.001 \\
\hline & & NM_019137 & Early growth response 4 & Egr4 & 11.8 & 0.001 \\
\hline & & NM_001107206 & AF4/FMR2 family, member 1 & Aff1 & 4.1 & 0.009 \\
\hline & & NM_144755 & Tribbles homolog 3 (Drosophila) & Trib3 & 4.7 & 0.008 \\
\hline & & NM_017334 & cAMP responsive element modulator variant 2 & Crem & 15.5 & 0.001 \\
\hline & & NM_145767 & Paired related homeobox protein-like 1 & Prrxl1 & -4.2 & 0.002 \\
\hline \multirow[t]{36}{*}{ G-2 } & \multirow{6}{*}{$\begin{array}{l}\text { GO:0016054 }(\mathrm{P}=0.001) \\
\text { organic acid catabolic } \\
\text { process }\end{array}$} & NM_145770 & Acyl-Coenzyme A oxidase 2 branched chain & Acox 2 & 14.2 & 0.002 \\
\hline & & NM_019168 & Arginase type II & $\operatorname{Arg} 2$ & 6.6 & 0.005 \\
\hline & & NM_053902 & Kynureninase & Kynu & 69.9 & 0.000 \\
\hline & & NM_013141 & Peroxisome proliferator-activated receptor delta & Ppard & 5.3 & 0.001 \\
\hline & & NM_138884 & Aldo-keto reductase family 1 member D1 & Akr1d1 & -10.6 & 0.003 \\
\hline & & NM_001012145 & Homogentisate 1, 2-dioxygenase & $\mathrm{Hgd}$ & -6.5 & 0.008 \\
\hline & \multirow{10}{*}{$\begin{array}{l}\text { GO:0055114 }(\mathrm{P}=0.002) \\
\text { oxidation reduction }\end{array}$} & NM_053433 & Flavin containing monooxygenase 3 & Fmo3 & 8.7 & 0.004 \\
\hline & & NM_001107295 & Oxidoreductase NAD-binding domain containing 1 & Oxnad 1 & 436.4 & 0.003 \\
\hline & & NM_012692 & Cytochrome P450, family 2 , subfamily a, polypeptide 1 & Cyp2a1 & -8.9 & 0.003 \\
\hline & & NM_012693 & Cytochrome P450, family 2 , subfamily a, polypeptide 2 & Cyp2a2 & -10.3 & 0.004 \\
\hline & & NM_019184 & Cytochrome P450, subfamily 2, polypeptide 11 & Cyp2c11 & -4.8 & 0.006 \\
\hline & & NM_019303 & Cytochrome P450, family 2, subfamily f, polypeptide 4 & Cyp2f4 & -4.5 & 0.003 \\
\hline & & NM_001135583 & Fatty acid 2-hydroxylase & $\mathrm{Fa} 2 \mathrm{~h}$ & -4.7 & 0.004 \\
\hline & & NM_012792 & Flavin containing monooxygenase 1 & Fmo1 & -5.0 & 0.000 \\
\hline & & NM_001009684 & Hydroxysteroid (17- $\beta$ ) dehydrogenase 13 & Hsd17b13 & -15.1 & 0.010 \\
\hline & & NM_012600 & Malic enzyme 1, NADP(+)-dependent, cytosolic & Me1 & -4.6 & 0.009 \\
\hline & \multirow{6}{*}{$\begin{array}{l}\text { GO:0003013 }(\mathrm{P}=0.005) \\
\text { circulatory system } \\
\text { process }\end{array}$} & NM_024483 & Adrenergic, alpha-1D $\mathrm{D}^{-}$, receptor & Adra1d & 6.5 & 0.002 \\
\hline & & NM_001102381 & Neurotensin & Nts & 4.1 & 0.007 \\
\hline & & NM_001007654 & Angiotensin II receptor-associated protein & Agtrap & -36.0 & 0.002 \\
\hline & & NM_031612 & Apelin & Apln & -14.1 & 0.001 \\
\hline & & NM_022936 & Epoxide hydrolase 2, cytoplasmic & Ephx2 & -29.0 & 0.002 \\
\hline & & NM_019160 & Urotensin 2 & Uts2 & -4.6 & 0.007 \\
\hline & \multirow{2}{*}{$\begin{array}{l}\text { GO:0005792 (P=0.001) } \\
\text { microsome }\end{array}$} & NM_012953 & Fos-like antigen 1 & Fosl1 & 8.5 & 0.010 \\
\hline & & NM_022280 & Lecithin retinol acyltransferase & Lrat & 7.6 & 0.007 \\
\hline & \multirow{12}{*}{$\begin{array}{l}\text { GO:0044421 }(\mathrm{P}=0.001) \\
\text { extra-cellular region part }\end{array}$} & NM_001107877 & ADAM with thrombospondin type 1 motif 9 & Adamts9 & 5.1 & 0.003 \\
\hline & & NM_012916 & Brevican, transcript variant 1 & Bcan & -4.9 & 0.006 \\
\hline & & XM_001066344 & Growth differentiation factor 5 & Gdf5 & 4.8 & 0.006 \\
\hline & & XM_213954 & Nidogen 1 & Nid1 & 4.7 & 0.007 \\
\hline & & NM_013151 & Plasminogen activator, tissue & Plat & 4.3 & 0.006 \\
\hline & & NM_001108533 & $\begin{array}{l}\text { Sparc/osteonectin, cwcv and kazal-like } \\
\text { domains proteoglycan } 2\end{array}$ & Spock2 & 8.9 & 0.001 \\
\hline & & NM_012881 & Secreted phosphoprotein 1 & Spp1 & 8.0 & 0.001 \\
\hline & & NM_013045 & Tenascin $\mathrm{R}$ & Tnr & 5.7 & 0.001 \\
\hline & & NM_019216 & Growth differentiation factor 15 & Gdf15 & -5.7 & 0.003 \\
\hline & & NM_001012741 & Lipase, endothelial & Lipg & -10.1 & 0.005 \\
\hline & & NM_001108356 & $\alpha$-fetoprotein & LOC360919 & -7.0 & 0.006 \\
\hline & & NM_001012027 & Serpin peptidase inhibitor, clade $\mathrm{C}$, member 1 & Serpinc1 & -593.4 & 0.000 \\
\hline
\end{tabular}


Table II. Continued.

\begin{tabular}{|c|c|c|c|c|c|c|}
\hline Group & GO-category & GenBank ID & Description & GS & $\mathrm{FC}$ & P-value \\
\hline & $\begin{array}{l}\text { GO:0020037 }(\mathrm{P}=0.004) \\
\text { heme binding }\end{array}$ & NM_001013853 & Globin, $\alpha$ & LOC287167 & -89.0 & 0.000 \\
\hline & $\begin{array}{l}\text { GO:0009055 }(\mathrm{P}=0.006) \\
\text { electron carrier activity }\end{array}$ & XM_001075627 & Cytochrome $c$ oxidase subunit VIIa-heart & LOC687508 & -4.9 & 0.002 \\
\hline & GO:0001653 (P=0.007) & NM_020542 & Chemokine (C-C motif) receptor 1 & Ccr1 & 4.4 & 0.010 \\
\hline & peptide receptor activity & NM_080411 & G protein-coupled receptor 83 & Gpr83 & 4.6 & 0.003 \\
\hline & & NM_198199 & Pyroglutamylated RFamide peptide receptor & Qrfpr & 4.1 & 0.001 \\
\hline & & NM_013064 & Hypocretin receptor 1 & Hcrtr1 & -4.7 & 0.009 \\
\hline \multirow[t]{17}{*}{ G-3 } & GO:0008217 (P=0.002) & NM_134432 & Angiotensinogen & Agt & 4.4 & 0.006 \\
\hline & regulation of blood & NM_001007654 & Angiotensin II receptor-associated protein & Agtrap & -29.6 & 0.000 \\
\hline & pressure & NM_022936 & Epoxide hydrolase 2, cytoplasmic & Ephx2 & -10.3 & 0.002 \\
\hline & & NM_019160 & Urotensin 2 & Uts2 & -13.1 & 0.001 \\
\hline & GO:0009891 (P=0.005) & NM_001106108 & Interferon regulatory factor 4 & Irf4 & 7.1 & 0.008 \\
\hline & positive regulation & XM_229993 & Cysteine-serine-rich nuclear protein 3 & Csrnp3 & -4.5 & 0.006 \\
\hline & of biosynthetic process & NM_019137 & Early growth response 4 & Egr4 & -5.2 & 0.004 \\
\hline & & NM_001108214 & Neuronal PAS domain protein 2 & Npas2 & -4.6 & 0.000 \\
\hline & & NM_031628 & $\begin{array}{l}\text { Nuclear receptor subfamily } 4 \text {, group A, member } 3 \text {, } \\
\text { transcript variant } 1\end{array}$ & $\mathrm{Nr} 4 \mathrm{a} 3$ & -7.6 & 0.001 \\
\hline & GO:0042592 (P=0.006) & NM_023969 & Lysophosphatidic acid receptor 3 & Lpar3 & 5.3 & 0.000 \\
\hline & homeostatic process & NM_001024767 & $\begin{array}{l}\text { Dual-specificity tyrosine-(Y)-phosphorylation } \\
\text { regulated kinase } 3\end{array}$ & Dyrk3 & -7.0 & 0.004 \\
\hline & & NM_212504 & Heat shock $70 \mathrm{kDa}$ protein $1 \mathrm{~B}$ & Hspa1b & -4.5 & 0.002 \\
\hline & & NM_001037357 & Leukocyte immunoglobulin-like receptor, subfamily B & Lilrb31 & -6.5 & 0.001 \\
\hline & & XM_001078539 & Ryanodine receptor type 1 Fragment & Ryr11 & -14.1 & 0.000 \\
\hline & GO:0019825 (P=0.003) & NM_012542 & Cytochrome P450, family 2 , subfamily a, polypeptide 3 & Cyp2a3 & -6.0 & 0.001 \\
\hline & oxygen binding & NM_019303 & Cytochrome P450, family 2 , subfamily f, polypeptide 4 & Cyp2f4 & -5.5 & 0.000 \\
\hline & & NM_001013853 & Globin, $\alpha$ & LOC287167 & -186.1 & 0.000 \\
\hline \multirow[t]{9}{*}{ G-4 } & GO:0045596 (P=0.003) & NM_024360 & Hairy and enhancer of split 1 (Drosophila) & Hes 1 & -7.0 & 0.005 \\
\hline & negative regulation & NM_133380 & Interleukin 4 receptor, alpha & Il4ra & -4.8 & 0.002 \\
\hline & of cell differentiation & NM_022392 & Insulin-induced gene 1 & Insig 1 & -4.7 & 0.000 \\
\hline & & NM_001107276 & Piwi-like 2 (Drosophila) & Piwil2 & -5.1 & 0.003 \\
\hline & & NM_001013181 & Zinc finger and BTB domain containing 16 & Zbtb16 & -4.7 & 0.002 \\
\hline & GO:0048545 $(\mathrm{P}=0.010)$ & NM_013157 & Argininosuccinate synthase 1 & Ass1 & 4.8 & 0.002 \\
\hline & response to steroid & NM_019160 & Urotensin 2 & Uts2 & 17.6 & 0.000 \\
\hline & hormone stimulus & NM_012953 & Fos-like antigen 1 & Fosl1 & -8.2 & 0.004 \\
\hline & & NM_175762 & Low density lipoprotein receptor & Ldlr & -7.4 & 0.000 \\
\hline
\end{tabular}

SHR- and SHRSP-specific genes were classified into 4 groups (Table I). The member of each group was further categorized into GO terms using DAVID web tools, and significantly enriched GO terms with $\mathrm{P}<0.01$ were identified. In the case where one gene was categorized into more than one GO term within the same group, one GO term was arbitrarily assigned to the gene. GS, gene symbol; FC, fold change of $>4$-fold upregulation and $<4$-fold downregulation; SHR, spontaneously hypertensive rats; SHRSP, stroke-prone SHR; GO, Gene Ontology.

the interaction between G-1 and G-2 genes, and found such a gene (Fos), which helps the interactions between $3 \mathrm{G}-1$ genes [Crem, Fosll and hematopoietically expressed homeobox (Hhex)] and many G-2 genes (Fig. 2). Among others, Crem seems to interact in the presence of Fos with genes regulating BP, such as Nts, Apln and Ephx2 (Fig. 2 and Table II, G-2), and with SHR-specific genes, such as Sppl, plasminogen activator, tissue (Plat) and aldo-keto reductase family 1 member D1 (Akrldl). Moreover, Crem indirectly interacted with many other SHR-specific genes, such as Adrald, chemokine (C-C motif) receptor 1 (Ccrl), Agtrap and Uts2 (Fig. 2). Although we did not find SHR-specific Fos transcripts among the transcripts of enriched G-1 and G-2 genes, we found that levels of the Fosll transcript in SHR at 3 and 6 weeks of age were 31.7- and 8.5-fold higher than those of the corresponding transcripts in WKY, respectively (Table II, G-1 and G-2). 


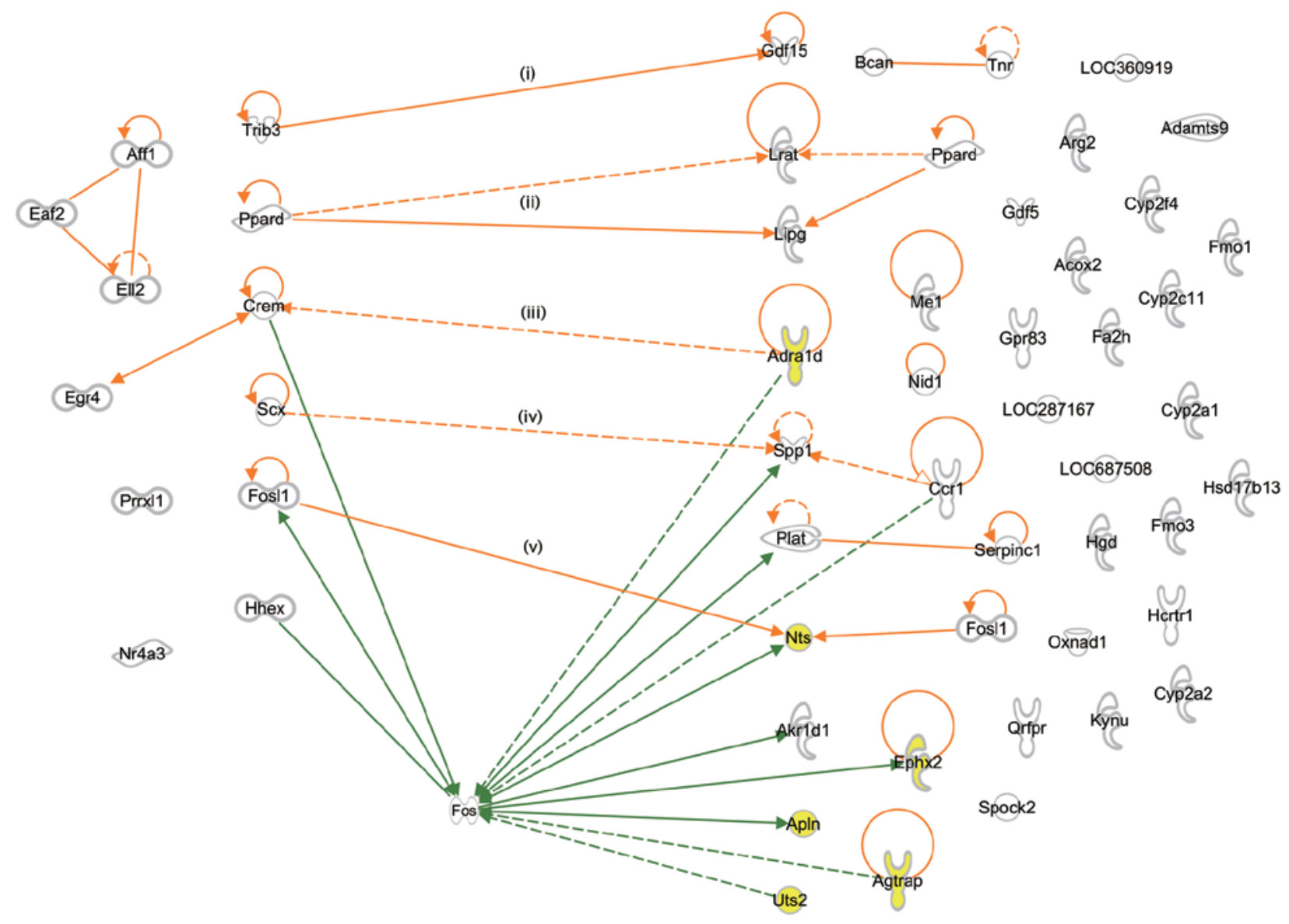

Figure 2. Analyses of interactions among SHR-specific genes. Interactions among SHR-specific genes isolated when the rats were 3 and 6 weeks of age were analyzed using Ingenuity Pathway Analysis (IPA). Depicted is the result of merging discovered gene-to-gene correlations. Genes are represented as nodes and the biological correlation between 2 nodes is represented as an edge (line). Edges signify different correlations as described below: solid lines represent direct interactions or associations and dotted lines represent indirect interactions or associations. Nodes are displayed using various shapes that represent the functional class of the gene product (refer to IPA for detailed node information). The 12 nodes (SHR-specific genes isolated at 3 weeks of age) on the left represent transcriptional regulator genes enriched by DAVID and the 42 nodes (SHR-specific genes isolated at 6 weeks of age) on the right represent genes enriched by DAVID. Interactions among SHR-specific genes are represented as orange edges and interactions between G-1 and G-2 genes are indicated by the numbers from (i) to (v). Interactions after postulating the presence of FOS are represented as green edges. BP-regulating genes categorized into GO:0003013 (circulatory system process), such as Adrald, Nts, Agtrap, Apln, Ephx2 and Uts2, are colored in yellow. SHR, spontaneously hypertensive rats; SHRSP, strokeprone SHR; WKY, Wistar-Kyoto rats.

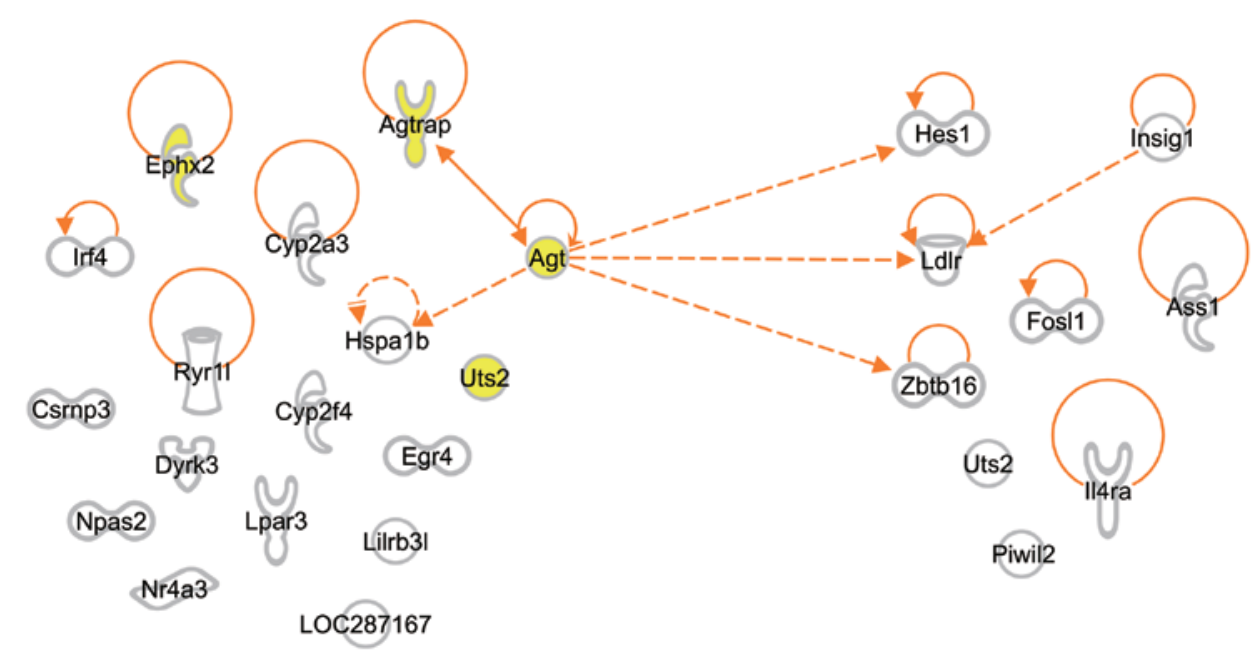

Figure 3. Analyses of interactions among SHRSP-specific genes. Interactions among SHRSP-specific genes isolated when the rats were 3 and 6 weeks of age were analyzed through the use of Ingenuity Pathway Analysis (IPA). The 17 nodes on the left correspond to enriched SHRSP-specific genes isolated when the rats were 3 weeks of age and the 9 nodes on the right correspond to enriched SHRSP-specific genes isolated when the rats were 6 weeks of age. BP-regulating SHRSP-specific genes isolated when the rats were 3 weeks of age and categorized into GO:0008217 (regulation of blood pressure), such as Agt, Agtrap, Ephx2 and $U t s 2$, are colored in yellow (see text). SHR, spontaneously hypertensive rats; SHRSP, stroke-prone SHR; WKY, Wistar-Kyoto rats. 
Fosll is a member of the Fos gene family, which consists of 4 members, Fos, Fosb, Fosll and Fosl2. Since Fosll has high Fos function rescue activity (21), we expect that Fosll replaces at least a part of Fos function and supports interactions between G-1 and G-2 genes (Fig. 2). Based on these observations, we propose that $\mathrm{Crem}$ is one of the candidate genes causing hypertension in SHR.

SHRSP-specific genes related to stroke-associated symptoms. Our results revealed that G-3 genes isolated from SHRSP at 3 weeks of age included a significant number of the genes isolated from SHR at 6 weeks of age, such as Uts2, Ephx2, Agtrap (GO:0008217, regulation of BP), cytochrome P450, family 2, subfamily f, polypeptide 4 (Cyp2f4) and globin, $\alpha$ (GloA) (GO:0019825, oxygen binding) (Table II). These results indicate that the evolution of the expression of genes related to BP control and to mitochondrial/cytochrome P450 systems proceed more rapidly in SHRSP than in SHR during their development.

We found that 4 of the 17 enriched G-3 genes, Agt, Agtrap, Ephx2 and Uts2, were isolated from SHRSP at 3 weeks of age and were categorized into GO:0008217 (regulation of BP): Agt was upregulated and the other 3 genes, Agtrap, Ephx2 and Uts2, were downregulated (Table II, G-3). Since the expression of these 4 genes was SHRSP-specific, we expected their participation in stroke-associated symptoms and examined the interactions between G-3 and G-4 genes by IPA. We found that Agt interacted not only with G-4 genes, such as Hes1, Zbtb16 and Ldlr, but also with G-3 genes, such as Agtrap and Hspalb (Fig. 3): Hes1 encodes a protein that belongs to the basic helix-loop-helix family of transcription factors and regulates transcription from RNA polymerase II promoter (22); Zbtb16 encodes a protein located in the nucleus and is involved in the positive regulation of transcription from RNA polymerase II promoter (23); and Ldlr mutations cause the autosomal dominant disorder, familial hypercholesterolemia $(24,25)$. Moreover, Agtrap encodes a protein that interacts with angiotensin II type I receptor and negatively regulates angiotensin II signaling (26) and Hspalb encodes a $70 \mathrm{kDa}$ heat shock protein that is a member of the heat shock protein 70 family and participates in the negative regulation of vasoconstriction (27). All these interactions suggest that $A g t$ plays pivotal roles in the pathogenesis of stroke.

Genes related to ADHD. SHR and SHRSP are frequently used as animal models in studies of ADHD $(10,11)$ and adrenal gland dysfunction is believed to be involved in ADHD due to low adrenaline (epinephrine) levels found in children with ADHD. Since juvenile SHRSP show significant increases in motor activity, one of the typical symptoms of ADHD as early as 6 weeks of age $(28,29)$, we expected that the expression levels of genes related to ADHD would show significant differences much earlier than 6 weeks of age and that SHRand SHRSP-specific genes isolated from the adrenal glands when the rats were 3 and 6 weeks of age not only include genes related to hypertension and stroke, but also include genes related to ADHD.

Genes involved in the metabolism and functions of corticosteroids are known to affect adrenaline levels in circulating blood and are differentially expressed in SHR or SHRSP.
For example, G-2 genes categorized into GO:0055114 (oxidation reduction), such as cytochrome P450 (Cyp)2al, Cyp2a2, Cyp2c11 and Cyp2f4 (Table II, G-2), and G-3 genes categorized into GO:0019825 (oxygen binding), such as Cyp2a3 and Cyp $2 f 4$ (Table II, G-3), catalyze many reactions involved in the synthesis of cholesterol, steroids and other lipids. Four of the G-4 genes, argininosuccinate synthase 1 (Assl), Uts2, Fosll and Ldlr, were categorized into GO:0048545 (response to a steroid hormone stimulus) (Table II, G-4). One of these genes, $L d l r$, is involved in the rate-limiting step in the synthesis of cholesterol and is reportedly related to hyperactive behavior (30).

In this study, we suggest that Crem is one of the candidate genes causing hypertension in SHR. Of note, Maldonado et al (31) reported that Crem-mutant mice exhibited behaviors similar to the symptoms observed in ADHD, such as an increased level of physical activity, as well as altered emotional and stress responses, and Lahti and Partonen (32) hypothesized that abnormalities in Crem protein functions or mutations in the Crem gene may underlie at least some of the symptoms in patients with ADHD.

Since functional and morphological studies in children affected by ADHD suggest not only adrenal gland dysfunctions, but also prefrontal cortex dysfunctions (33), we extended our current study to examine gene expression profiles in brains derived from SHR and SHRSP at 3 and 6 weeks of age.

In conclusion, SHR and SHRSP are widely used as animal models, not only in studies of essential hypertension, but also in studies of ADHD. Using these animal models, in the present study, 12 enriched SHR-specific genes exhibiting transcriptional regulatory activity were isolated from the adrenal glands when the rats were 3 weeks of age and one of these 12 genes, $\mathrm{Crem}$, was suggested to be a possible candidate gene causing hypertension in SHR. Similarly, our results suggest that Agt plays pivotal roles in causing stroke. Genes involved in ADHD were also discussed.

\section{Acknowledgements}

We thank Dr Etsuro Yamanishi, President Emeritus of Hirakata General Hospital for Developmental Disorders, and Dr Aritomo Suzuki, Professor Emeritus of Kinki University, for their constant support and encouragement, and thank Miss Fumie Kanazawa for her expert secretarial assistance. We also thank the National Center for Biotechnology Information, US National Library of Medicine, Bethesda, MD, USA and the DNA Data Bank of Japan for access to network servers.

\section{References}

1. McBride MW, Charchar FJ, Graham D, Miller WH, Strahorn P, Carr FJ and Dominiczak AF: Functional genomics in rodent models of hypertension. J Physiol 554: 56-63, 2004.

2. Delles C, McBride MW, Graham D, Padmanabhan S and Dominiczak AF: Genetics of hypertension: from experimental animals to humans. Biochim Biophys Acta 1802: 1299-1308, 2010.

3. Okamoto K and Aoki K: Development of a strain of spontaneously hypertensive rats. Jpn Circ J 27: 282-293, 1963.

4. Okamoto K, Hazama F, Yamori Y, Haebara H and Nagaoka A: Pathogenesis and prevention of stroke in spontaneously hypertensive rats. Clin Sci Mol Med (Suppl) 2: 161s-163s, 1975.

5. Nabika T, Cui Z and Masuda J: The stroke-prone spontaneously hypertensive rat: how good is it as a model for cerebrovascular diseases? Cell Mol Neurobiol 24: 639-646, 2004. 
6. Friese RS, Mahboubi P, Mahapatra NR, et al: Common genetic mechanisms of blood pressure elevation in two independent rodent models of human essential hypertension. Am J Hypertens 18: 633-652, 2005.

7. Ashenagar MS, Tabuchi M, Kinoshita K, et al: Gene expression in the adrenal glands of three spontaneously hypertensive rat substrains. Mol Med Rep 3: 213-222, 2010.

8. Huang da W, Sherman BT and Lempicki RA: Systematic and integrative analysis of large gene lists using DAVID bioinformatics resources. Nat Protoc 4: 44-57, 2009.

9. Huang da W, Sherman BT and Lempicki RA: Bioinformatics enrichment tools: paths toward the comprehensive functional analysis of large gene lists. Nucleic Acids Res 37: 1-13, 2009

10. Sagvolden T, Russell VA, Aase H, Johansen EB and Farshbaf M: Rodent models of attention-deficit/hyperactivity disorder. Biol Psychiatry 57: 1239-1247, 2005.

11. Faraone SV and Mick E: Molecular genetics of attention deficit hyperactivity disorder. Psychiatr Clin North Am 33: 159-180, 2010.

12. Kempf T, Eden M, Strelau J, et al: The transforming growth factor-beta superfamily member growth-differentiation factor-15 protects the heart from ischemia/reperfusion injury. Circ Res 98 : 351-360, 2006

13. de Jager SC, Bermúdez B, Bot I, et al: Growth differentiation factor 15 deficiency protects against atherosclerosis by attenuating CCR2-mediated macrophage chemotaxis. J Exp Med 208: 217-225, 2011.

14. Liu L and Gudas LJ: Disruption of the lecithin:retinol acyltransferase gene makes mice more susceptible to vitamin A deficiency. J Biol Chem 280: 40226-40234, 2005.

15. Qiu G and Hill JS: Atorvastatin decreases lipoprotein lipase and endothelial lipase expression in human THP-1 macrophages. J Lipid Res 48: 2112-2122, 2007.

16. García-Cazarín ML, Smith JL, Clair DK and Piascik MT: The alpha1D-adrenergic receptor induces vascular smooth muscle apoptosis via a p53-dependent mechanism. Mol Pharmacol 74 1000-1007, 2008.

17. Denhardt DT and Guo X: Osteopontin: a protein with diverse functions. FASEB J 7: 1475-1482, 1993.

18. Wang KX and Denhardt DT: Osteopontin: role in immune regulation and stress responses. Cytokine Growth Factor Rev 19: 333-345, 2008

19. Reghunandanan V and Reghunandanan R: Neurotransmitters of the suprachiasmatic nuclei. J Circadian Rhythms 4: 2, 2006.

20. Roubert C, Spielewoy C, Soubrié P, Hamon M, Giros B and Betancur C: Altered neurotensin mRNA expression in mice lacking the dopamine transporter. Neuroscience 123: 537-546, 2004
21. Matsuo K, Owens JM, Tonko M, Elliott C, Chambers TJ and Wagner EF: Fosl1 is a transcriptional target of c-Fos during osteoclast differentiation. Nat Genet 24: 184-187, 2000.

22. Hirata H, Tomita K, Bessho Y and Kageyama R: Hes1 and Hes3 regulate maintenance of the isthmic organizer and development of the mid/hindbrain. EMBO J 20: 4454-4466, 2001.

23. Senbonmatsu T, Saito T, Landon EJ, et al: A novel angiotensin II type 2 receptor signaling pathway: possible role in cardiac hypertrophy. EMBO J 22: 6471-6482, 2003.

24. Scotti E, Hong C, Yoshinaga Y, et al: Targeted disruption of the idol gene alters cellular regulation of the low-density lipoprotein receptor by sterols and liver $\mathrm{x}$ receptor agonists. Mol Cell Biol 31: 1885-1893, 2011.

25. Hartvigsen K, Chou MY, Hansen LF, Shaw PX, Tsimikas S, Binder CJ and Witztum JL: The role of innate immunity in atherogenesis. J Lipid Res 50 (Suppl): S388-S393, 2009.

26. Azuma K, Tamura K, Shigenaga A, et al: Novel regulatory effect of angiotensin II type 1 receptor-interacting molecule on vascular smooth muscle cells. Hypertension 50: 926-932, 2007.

27. Zitvogel L, Kepp O and Kroemer G: Decoding cell death signals in inflammation and immunity. Cell 140: 798-804, 2010.

28. Ueno KI, Togashi H, Mori K, et al: Behavioural and pharmacological relevance of stroke-prone spontaneously hypertensive rats as an animal model of a developmental disorder. Behav Pharmacol 13: 1-13, 2002.

29. Anderson GM, Dover MA, Yang BP, et al: Adrenomedullary function during cognitive testing in attention-deficit/hyperactivity disorder. J Am Acad Child Adolesc Psychiatry 39: 635- 643, 2000

30. Mack JT, Beljanski V, Soulika AM, Townsend DM, Brown CB, Davis W and Tew KD: 'Skittish' Abca2 knockout mice display tremor, hyperactivity, and abnormal myelin ultrastructure in the central nervous system. Mol Cell Biol 27: 44-53, 2007.

31. Maldonado R, Smadja C, Mazzucchelli C and Sassone-Corsi P: Altered emotional and locomotor responses in mice deficient in the transcription factor CREM. Proc Natl Acad Sci USA 96: 14094-14099, 1999.

32. Lahti TA and Partonen T: CREM mutations and ADHD symptoms. Med Hypotheses 72: 544-545, 2009.

33. Viggiano D, Ruocco LA, Arcieri S and Sadile AG: Involvement of norepinephrine in the control of activity and attentive processes in animal models of attention deficit hyperactivity disorder. Neural Plast 11: 133-149, 2004 